\title{
RBP3: a possible prognostic marker and therapeutic target in diabetic retinopathy
}

\author{
Dario Rusciano', Paola Bagnoli ${ }^{2}$ \\ ${ }^{1}$ SOOFT Italia SpA, Research Department c/o Biologic Tower, University of Catania, Catania, Italy; ${ }^{2}$ Biology Department, University of Pisa, Pisa, \\ Italy \\ Correspondence to: Dario Rusciano. Sooft Italia SpA, c/o Torre Biologica, University of Catania, Via Santa Sofia 89, 95123 Catania, Italy. \\ Email: dario.rusciano@sooft.it. \\ Provenance: This is an invited article commissioned by the Section Editor Dr. Wan Wang (Medical Technology School, Xuzhou Medical University, \\ Xuzhou, China). \\ Comment on: Yokomizo H, Maeda Y, Park K, et al. Retinol binding protein 3 is increased in the retina of patients with diabetes resistant to diabetic \\ retinopathy. Sci Transl Med 2019. doi: 10.1126/scitranslmed.aau6627.
}

Submitted Aug 31, 2019. Accepted for publication Sep 26, 2019.

doi: 10.21037/atm.2019.09.133

View this article at: http://dx.doi.org/10.21037/atm.2019.09.133

Is RBP3 just another common piece in the puzzle of diabetic retinopathy, or could it be a key card for its understanding and maybe even treatment?

Chronic progressive pathologies, such as diabetes, are hard to manage and treat, because too little is known of their etiology (partly genetic, partly environmental); early diagnosis can be hard to achieve, and reliable specific prognostic markers are missing, with the exception of a generic index of the glycemic control such as glycosylated hemoglobin (HbAc). In fact, blood sugar control and insulin therapy is mostly all that is available nowadays to manage diabetes and decrease the risk of one of its most devastating consequences: proliferative diabetic retinopathy. PDR is among the main causes of blindness in the western world, and sure enough a deeper understanding of its molecular pathology will be useful to increase our ability to follow and maybe treat this disease. Several decades of research in this field have generated a tree of risk factors and some biomarkers that are variably associated with the disease and its progression. Arterial hypertension (1), oxydative stress (2), longer duration of diabetes mellitus, type $1 v s .2$ of diabetes mellitus, bad metabolic control (i.e., high HbA1c level), and shorter axial length (hyperopia) are the accepted risk factors (3). The inflammatory events that follow the metabolic dysregulation may lead to the accumulation of inflammatory cytokines in the vitreous body (4), triggering autophagy and apoptosis in retinal pigment epithelial cells (RPE), essential to photoreceptor survival (5). Metabolic profiling of blood or vitreous body of PDR patients have indicated several biomarkers associated with disease progression $(6,7)$. The presence of specific microRNAs has been linked to PDR evolution $(8,9)$. However, none of the above elements can be reliably used as individual prognostic marker, even less as a specific therapeutic target. More recently, angiopoietin-like 3 (ANGPTL3) has been described as independently and strongly associated with DR progression in all stages. Therefore, blockade of ANGPTL3 signal in retina might postpone the onset and development of DR in type 2 diabetic patients (10). However, no proof of concept exists as yet to this inference. With a different strategy, our research group has exploited the anti-angiogenic and antinflammatory properties of a synthetic peptide (UPARANT: Urokinase Plasminogen Activator Receptor ANTagonist) designed to interfere with the downstream signaling of the formyl peptide receptor (FPR) and associated proteins, to partially rescue the retinal degeneration in animal model systems of type I (11) and type II (12) diabetes, thus showing the relevance of the UPAR/FPR system in the vascular dysregulation occurring during diabetes progression and DR (13).

Despite intensive research into most aspects of metabolic diseases including diabetes mellitus and its complications, much work remains to be done to identify accessible predictive biomarkers that can guide the pharmacological treatment. In this respect, most research groups are directing their studies toward the identification of potential 
circulating biomarkers with precise prognostic value (14). Several molecular biomarkers show promise as screening markers to detect early diabetic retinopathy or even to detect patients at increased risk of diabetic retinopathy at the time of diagnosis of diabetes. Among molecular tools, scientists have recently shifted their research focus towards non coding RNA molecules with the aim of developing novel specific biomarkers for early detection of metabolic disorders and their complications. Little is known on the functional properties of non coding RNAs although there are some indications that they may interact with RNA-binding proteins (RBPs) to contribute to the pathogenesis of diverse metabolic processes including their ophthalmological complications (15). In diabetic retinopathy, for instance, there are some findings indicating that non coding RNAs participate to the regulation of endothelial cell function through major effects on retinal levels of VEGF (16). Although much work remains to be done to clarify the role of non coding RNAs as sensors and biomarkers for metabolic disorders, the possibility to correlate their plasmatic levels with the severity of the disease remains very exciting.

In the context of predictive biomarkers a recent paper of Yokomizo et al. (17) based on previous work done by other groups (18-20), emphasized the importance of the retinol binding protein RBP3 (exclusively produced by retina photoreceptors) as differentially present in the retina and the vitreous humor of mostly type I diabetic patients affected by different degrees of DR. High amounts of RBP3 in the retina and the vitreous correlated with no or low grade DR, low amounts with the presence of PDR. Most strikingly, artificially induced overexpression of RBP3 in vivo or in vitro attenuated $\mathrm{DR}$ and its related events. A mechanism by which RBP3 appears to exert its effects is RBP3 antagonistic binding to GLUT1, a major glucose transporter through the blood-retinal barrier. GLUT1 has been identified as a promising target for DR since the inhibition of its expression decreases the retinal glucose concentration in diabetic mice and ameliorates the pathological signs of diabetic retinopathy (21). The overexpression of RBP3 would lead to the inhibition of glucose uptake in retinal cells with subsequent decreased expression of VEGF and inflammatory cytokines (17). Therefore, RBP3 appears to be not only another biomarker associated with DR, but a key player in its development and progression, and a possible element to exploit in therapy.

Too good to be true? In fact, in order to bring this discovery from the bench to the bedside, as translational research is supposed to do, there are still some relevant matters to be better elucidated.

RBP3 is found in the retina and the vitreous; only a tiny amount of unknown origin is present in the blood (though detectable by a high-sensitivity ELISA). In order to be used as a prognostic biomarker in living individuals, RBP3 must be detected in accessible periferal fluids. Therefore, it is important to know what is its distribution in the population: whether the high-sensitivity ELISA will allow to identify and discriminate groups with different serum concentrations of RBP3, and how stable in time is such asset: is it age and/or life-style dependent? And this leads to another key question: are the different amounts of RBP3 found in the retina and the vitreous a cause or an effect of DR progression? If individuals were endowed with different expression abilities of RBP3, it might be plausible that its molecular functions as defined by the study of Yokomizo et al. may protect the retina from degenerative events, conferring to their possessors a higher resistance to DR progression. On the other hand, if RBP3 were found to be expressed at similar levels in all individuals, so that the initial degree of protection is comparable, then it could be that its susceptibility to degradation is different. In this case, RBP3 would be less degraded (and more protective) in the slow-progressors, and more degraded (and less protective) in fast progressors, so that patients with PDR have less residual RBP3 and more degradation products, as data in this paper might suggest. This hypothetical different resistance of RBP3 to degradation might depend on allelic variations of the protein, or on upstream events impinging on RBP3 stability.

Another key point is whether in diabetic patients different serum concentrations of RBP3 may also represent a risk factor for progression. Indeed, it could already be possible from the data obtained in this published study to see whether there is a hint of correlation between RBP3 concentration in serum and in the eye, and therefore with the likelihood of PDR. In such case, a prospective study would be feasible, to address the question as to whether progression of DR in an early diabetic population correlates to RBP3 expression. Alternatively, expression genetics of RBP3 and its allelic variants (if any) could be addressed, with the same goal of validating RBP3 as a prognostic marker. However, even though it could not be validated as such, the observation remains that RBP 3 overexpression is protective against $\mathrm{DR}$, and therefore it could be a tool to be exploited to delay or soften the adverse events of long-term diabetes and hyperglycemia. To this purpose, the regulation 
of RBP3 expression could be addressed, in order to find strategies - if possible- to enhance its expression by means of natural or artificial drugs.

In the past, another retinol binding protein (RBP4) has been linked to diabetes and retinal degeneration, in an opposite way to RBP3 $(22,23)$. RBP4 is an adipocytederived 'signal' that may contribute to the pathogenesis of type 2 diabetes (24). In fact, serum RBP4 levels are elevated in insulin-resistant mice and humans with obesity and type 2 diabetes. RBP4 circulates in the plasma bound to its carrier protein transthyretin (TTR), which prevents its clearance through the kidneys (25). Its expression appears to be linked to that of the glucose transporter GLUT4. Transmembrane transport of glucose by GLUT4 is the rate-limiting factor for glucose transport in adipose tissue and skeletal muscle and downregulation of GLUT4 expression is characteristic of insulin-resistant states, including obesity, type 2 diabetes and the metabolic syndrome. RBP4 expression increases when GLUT4 expression goes down (26,27). Therefore, it appears that the axes RBP4/GLUT4 and RBP3/GLUT1 work in different ways with opposing results: lowering of RBP4 could be a strategy for treating type 2 diabetes (and indeed, as a proof of concept, a non-retinoid antagonist of RBP4 has been shown to rescue the phenotype in a model of Stargardt disease (28), whereas it is an upregulation of the axis RBP3/GLUT1 which could be beneficial to type I (and maybe also type II) diabetes. The relationship between RBP3 and TTR, if any, remains to be elucidated.

In conclusion, RBP3 appears to have the characteristics of a turning point in the understanding of DR and its progression; whether it will turn out to be a critical milestone, or just another cobblestone remains to be shown.

\section{Acknowledgments}

Funding: Part of the authors' research has been supported by grants from the Italian Ministero della Salute (RF201102351158; PB; Roma, Italy), Italian Ministero dell'Istruzione e dell'Università and the European Community (PON01 02464).

\section{Footnote}

Conflicts of Interest: The authors have no conflicts of interest to declare.

Ethical Statement: The authors are accountable for all aspects of the work in ensuring that questions related to the accuracy or integrity of any part of the work are appropriately investigated and resolved.

\section{References}

1. Pescosolido N, Rusciano D. Diabetic Retinopathy and Hypertension. Ann Clin Exp Hypertens 2015;3:1022.

2. Nebbioso M, Federici M, Rusciano D, et al. Oxidative stress in preretinopathic diabetes subjects and antioxidants. Diabetes Technol Ther 2012;14:257-63.

3. Jonas JB, Sabanayagam C. Epidemiology and Risk Factors for Diabetic Retinopathy. In: Sabanayagam C, Wong TY. editors. Diabetic Retinopathy and Cardiovascular Disease. Basel: Karger, 2019;27:20-37.

4. Rezzola S, Corsini M, Chiodelli P, et al. Inflammation and $\mathrm{N}$-formyl peptide receptors mediate the angiogenic activity of human vitreous humour in proliferative diabetic retinopathy. Diabetologia 2017;60:719-28.

5. Rosa MD, Distefano G, Gagliano C, et al. Autophagy in Diabetic Retinopathy. Curr Neuropharmacol 2016;14:810-25.

6. Zhu XR, Yang FY, Lu J, et al. Plasma metabolomic profiling of proliferative diabetic retinopathy. Nutr Metab (Lond) 2019;16:37.

7. Sahajpal NS, Vig VK, Singh R, et al. Deranged Metabolic Profile and Identification of Biomarkers in the Vitreous Humour of Patients with Proliferative Diabetic Retinopathy. Protein Pept Lett 2018;25:791-8.

8. Martinez B, Peplow PV. MicroRNAs as biomarkers of diabetic retinopathy and disease progression. Neural Regen Res 2019;14:1858-69.

9. Pastukh N, Meerson A, Kalish D, et al. Serum miR-122 levels correlate with diabetic retinopathy. Clin Exp Med 2019;19:255-60.

10. Yu CG, Yuan SS, Yang LY, et al. Angiopoietin-like 3 Is a Potential Biomarker for Retinopathy in Type 2 Diabetic Patients. Am J Ophthalmol 2018;191:34-41.

11. Cammalleri M, Locri F, Marsili S, et al. The Urokinase Receptor-Derived Peptide UPARANT Recovers Dysfunctional Electroretinogram and Blood-Retinal Barrier Leakage in a Rat Model of Diabetes. Invest Ophthalmol Vis Sci 2017;58:3138-48.

12. Cammalleri M, Dal Monte M, Locri F, et al. Diabetic Retinopathy in the Spontaneously Diabetic Torii Rat: Pathogenetic Mechanisms and Preventive Efficacy of Inhibiting the Urokinase-Type Plasminogen Activator Receptor System. J Diabetes Res 2017;2017:2904150.

13. Cammalleri M, Dal Monte M, Pavone V, et al. The uPAR 
System as a Potential Therapeutic Target in the Diseased Eye. Cells 2019. doi: 10.3390/cells8080925.

14. Simó-Servat O, Simó R, Hernández C. Circulating Biomarkers of Diabetic Retinopathy: An Overview Based on Physiopathology. J Diabetes Res 2016;2016:5263798.

15. Zhang L, Dong Y, Wang Y, et al. Long non-coding RNAs in ocular diseases: new and potential therapeutic targets. FEBS J 2019;286:2261-72.

16. Thomas AA, Feng B, Chakrabarti S. ANRIL: A Regulator of VEGF in Diabetic Retinopathy. Invest Ophthalmol Vis Sci 2017;58:470-80.

17. Yokomizo H, Maeda Y, Park K, et al. Retinol binding protein 3 is increased in the retina of patients with diabetes resistant to diabetic retinopathy. Sci Transl Med 2019. doi: 10.1126/scitranslmed.aau6627.

18. Garcia-Ramírez M, Hernández C, Villarroel M, et al. Interphotoreceptor retinoid-binding protein (IRBP) is downregulated at early stages of diabetic retinopathy. Diabetologia 2009;52:2633-41.

19. Arno G, Hull S, Robson AG, et al. Lack of Interphotoreceptor Retinoid Binding Protein Caused by Homozygous Mutation of RBP3 Is Associated With High Myopia and Retinal Dystrophy. Invest Ophthalmol Vis Sci 2015;56:2358-65.

20. Liu YH, Corbett C, Klaska IP, et al. Partial retinal photoreceptor loss in a transgenic mouse model associated with reduced levels of interphotoreceptor retinol binding protein (IRBP, RBP3). Exp Eye Res 2018;172:54-65.

21. You ZP, Zhang YL, Shi K, et al. Suppression of diabetic

Cite this article as: Rusciano D, Bagnoli P. RBP3: a possible prognostic marker and therapeutic target in diabetic retinopathy. Ann Transl Med 2019;7(Suppl 8):S327. doi: 10.21037/atm.2019.09.133 retinopathy with GLUT1 siRNA. Sci Rep 2017;7:7437.

22. Yang Q, Graham TE, Mody N, et al. Serum retinol binding protein 4 contributes to insulin resistance in obesity and type 2 diabetes. Nature 2005;436:356-62.

23. Du M, Otalora L, Martin AA, et al. Transgenic Mice Overexpressing Serum Retinol-Binding Protein Develop Progressive Retinal Degeneration through a RetinoidIndependent Mechanism. Mol Cell Biol 2015;35:2771-89.

24. Kelly KR, Kashyap SR, O'Leary VB, et al. Retinol-binding protein 4 (RBP4) protein expression is increased in omental adipose tissue of severely obese patients. Obesity (Silver Spring) 2010;18:663-6.

25. Naylor HM, Newcomer ME. The structure of human retinol-binding protein (RBP) with its carrier protein transthyretin reveals an interaction with the carboxy terminus of RBP. Biochemistry 1999;38:2647-53.

26. Majerczyk M, Olszanecka-Glinianowicz M, PuzianowskaKuźnicka M, et al. Retinol-binding protein 4 (RBP4) as the causative factor and marker of vascular injury related to insulin resistance. Postepy Hig Med Dosw (Online) 2016;70:1267-75.

27. Smith U, Kahn BB. Adipose tissue regulates insulin sensitivity: role of adipogenesis, de novo lipogenesis and novel lipids. J Intern Med 2016;280:465-75.

28. Racz B, Varadi A, Kong J, et al. A non-retinoid antagonist of retinol-binding protein 4 rescues phenotype in a model of Stargardt disease without inhibiting the visual cycle. J Biol Chem 2018;293:11574-88. 